\title{
Bottom-Up Earley Deduction
}

\author{
Gregor Erbach* \\ University of the Saarland \\ Computational Linguistics \\ D-66041 Saarbrücken, Germany \\ erbach@coli.uni-sb.de
}

\begin{abstract}
We propose a bottom-up variant of Earley deduction. Bottom-up deduction is preferable to top-down deduction because it allows incremental processing (even for head-driven grammars), it is data-driven, no subsumption check is needed, and preference values attached to lexical items can be used to guide best-first search. We discuss the scanning step for bottom-up Earley deduction and indexing schemes that help avoid useless deduction steps.
\end{abstract}

\section{Introduction}

Recently, there has been a lot of interest in Earley deduction [10] with applications to parsing and generation $[13,6,7,3]$.

Earley deduction is a very attractive framwork for natural language processing because it has the following properties and applications.

- Memoization and reuse of partial results

- Incremental processing by addition of new items

- Hypothetical reasoning by keeping track of dependencies between items

- Best-first search by means of an agenda

*This work was supported by the Deutsche Forschungsgemeinschaft through the project N3 "Bidirektionale Linguistische Deduktion (BiLD)" in the Sonderforschungsbereich 314 Künstliche Intelligenz - Wissensbasierte Systeme and by the Commission of the European Communities through the project I,RE-61-061 "Reusable Grammatical Resources." I would like to thank Günter Neumann, Christer Samuelsson and Mats Wirén for comments on this paper.
Like Earley's algorithm, all of these approaches operate top-down (backward chaining). The interest has naturally focussed on top-down methods because they are at least to a certain degree goal-directed.

In this paper, we present a bottom-up variant of Earley deduction, which we find advantageous for the following reasons:

Incrementality: Portions of an input string can be analysed as soon as they are produced (or generated as soon as the what-to-say component has decided to verbalize them), even for grammars where one cannot assume that the left-corner has been predicted before it is scanned.

Data-Driven Processing: Top-down algorithms are not well suited for processing grammatical theories like Categorial Grammar or HPSG that would only allow very general predictions because they make use of general schemata instead of constructionspecific rules. For these grammars datadriven bottom-up processing is more appropriate. The same is true for large-coverage rule-based grammars which lead to the creation of very many predictions.

Subsumption Checking: Since the bottom-up algorithm does not have a prediction step, there is no need for the costly operation of subsumption checking. ${ }^{1}$

Search Strategy: In the case where lexical entries have been associated with preference in-

\footnotetext{
${ }^{1}$ Subsumption checking may still be needed to filter out spurious ambiguities.
} 
formation, this information can be exploited to guide the heuristic search.

\section{Bottom-up Earley Deduction}

Farley deduction [10] is based on grammars encoded as definite clauses. The instantiation (prediction) rule of top-down Earley deduction is not needed in bottom up Farley deduction, because there is no prediction. 'There is only one inference rule, namely the reduction rule (1). ${ }^{2}$ In (1), $X$, $G$ and $G^{\prime}$ are literals, $\Omega$ is a (possibly empty) sequence of literals, and $\sigma$ is the most general unifier of $G$ and $G^{\prime}$. The leftmost literal in the body of a non-unit clause is always the selected literal.

$$
\begin{gathered}
X \leftarrow G \wedge \Omega \\
G^{\prime} \leftarrow \\
\hline \sigma(X \leftarrow \Omega)
\end{gathered}
$$

In principle, this rule can bo applied to any pair of nuit clanses and non-unit clauses of the program to derive any consequences of the program. In order to reduce this search space and achicve a more goal-directed behaviont, the rule is not applied to any pair of clanses, but clauses are only selected if they can contribute to a proof of the goal. 'The set of selected clauses is called the chart. ${ }^{3}$ The selection of clanses is guided by a scanning step (section 2.1) and indexing of clauses (section 2.2).

\subsection{Scanning}

The purpose of the scanning step, which corresponds to lexical lookup in chart parsers, is to look up base cases of recursive definitions to serve as a staring point for bottom-up processing. 'The scanning step selects clauses that can appear as leaves in the proof tree for a given goal $C$.

Consider the following simple definition of an HPSG, with the recursive definition of the predicale sign/1.4

\footnotetext{
${ }^{2}$ This rule is called combine by Earley, and is also referred to as the fundanental rule in the literature on chart parsing.

"The chart differs from the state of $[10]$ in that clauses in the chart are indexed (cf. section 2.2).

${ }^{A}$ We use feature terms in definite clauses in addition to Prolog terins. $\mathbf{f}: \mathrm{X}$ denotes a feature structure where $\mathrm{X}$ is the value of feature $f$, and $X \& Y$ denotes the conjunction
}

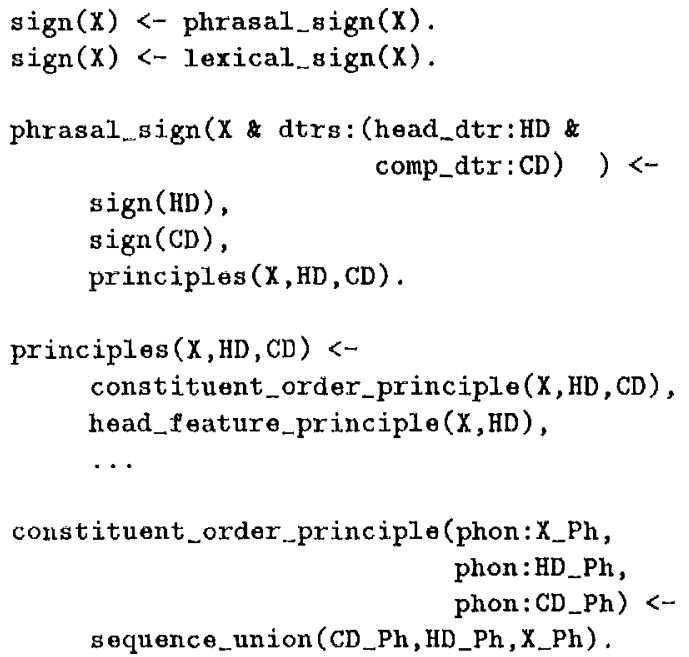

The predicate sign/1 is defined recursively, and the base case is the predicate lexical_sign/1. But, clearly it is not restrictive enough to find only the predicate name of the base case for a given goal. 'The base cases must also be instantiated in order to find those that are useful for proving a given goal. In the case of parsing, the lookup of base cases (lexical items) will depend on the words that are present in the input string. This is implied by the first goal of the predicate principles/3, the constituent order principle, which determines how the pHON value of a constituent is constructed from the proon values of its daughters. In general, we assume that the constituent order principle makes use of a linear and non-erasing operation for combining strings. ${ }^{5}$ If this is the case, then all the words contained in the Pron value of the goal can have their lexical items selected as unit clauses to start bottom-up processing.

For gencration, an analogous condition on logical forms has been proposed by Shieber [13] as the "semantic monotonicity condition," which requires that the logical form of every base case must subsume some portion of the goal's logical form.

Base case lookup must be defined specifically for different grammatical theories and directions of processing by the predicate lookup/2, whose first argument is the goal and whose second argument is the selected base case. The following

of the feature terms $X$ and $Y$.

"There is an obvious connection to the Linear ContextFree Rewriting Systems (LCFRS) $[15,16]$. 
clause defines the lookup relation for parsing with HPSG.

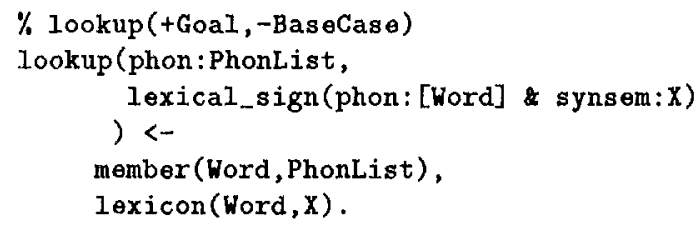

Note that the base case clauses can become further instantiated in this step. If concatenation (of difference lists) is used as the operation on strings, then each base case clause can be instantiated with the string that follows it. This avoids combination of items that are not adjacent in the input string.

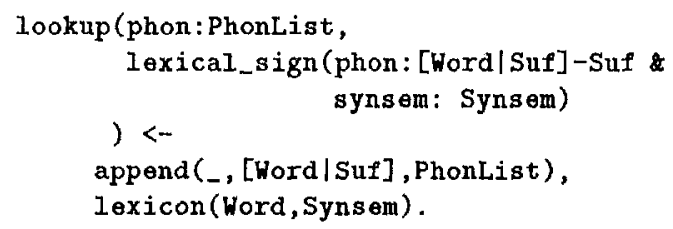

In bottom-up Earley deduction, the first step towards proving a goal is perform lookup for the goal, and to add all the resulting (unit) clauses to the chart. Also, all non-unit clauses of the program, which can appear as internal nodes in the proof tree of the goal, are added to the chart.

The scanning step achieves a certain degree of goal-directedness for bottom-up algorithms because only those clauses which can appear as leaves in the proof tree of the goal are added to the chart.

\subsection{Indexing}

An item in normal context-free chart parsing can be regarded as a pair $\langle R, S\rangle$ consisting of a dotted rule $R$ and the substring $S$ that the item covers (a pair of starting and ending position). The fundamental rule of chart parsing makes use of these string positions to ensure that only adjacent substrings are combined and that the result is the concatenation of the substrings.

In grammar formalisms like DCG or H PSG, the complex nonterminals have an argument or a feature (PHON) that represents the covered substring explicitly. The combination of the substrings is explicit in the rules of the grammar. As a consequence, Earley deduction does not need to make use of string positions for its clauses, as Pereira and Warren [10] point out.

Moreover, the use of string positions known from chart parsing is too inflexible because it allows only concatenation of adjacent contiguous substrings. In linguistic theory, the interest has shifted from phrase structure rules that combine adjacent and contiguous constituents to

- principle-based approaches to grammar that state general well-formedness conditions instead of describing particular constructions (e.g. HPSG)

- operations on strings that go beyond concatenation (head wrapping [11], tree adjoining [15], sequence union [12]).

The string positions known from chart parsing are also inadequate for generation, as pointed out by Shieber [13] in whose generator all items go from position 0 to 0 so that any item can be combined with any item.

However, the string positions are useful as an indexing of the items so that it can be easily detected whether their combination can contribute to a proof of the goal. This is especially important for a bottom-up algorithm which is not goal-directed like top-down processing. Without indexing, there are too many combinations of items which are useless for a proof of the goal, in fact there may be infinitely many items so that termination problems can arise.

For example, in an order-monotonic grammar formalism that uses sequence union as the operation for combining strings, a combination of items would be useless which results in a sign in which the words are not in the same order as in the input string [14].

We generalize the indexing scheme from chart parsing in order to allow different operations for the combination of strings. Indexing improves efficiency by detecting combinations that would fail anyway and by avoiding combinations of items that are useless for a proof of the goal.

We define an item as a pair of a clause $C l$ and an index $I d x$, written as $\langle C l, I d x\rangle$. 
Below, we give some examples of possible indexing schemes. Other indexing schemes can be used if they are needed.

1. Non-reuse of Items: This is useful for IJCRRS, where no word of the input string can be used twice in a proof, or for gencration where no part of the goal logical form should be verbalized twice in a derivation.

2. Non-adjacent combination: 'l'his indexing scheme is useful for order-monotonic grammars.

\section{Non-directional adjacent combination:} This indexing is used if only adjacent constituents can be combined, but the order of combination is not prescribed (e.g. nondirectional basic categorial grammars).

\section{Directional adjacent combination:}

This is used for grammars with a "contextfree backbone."

5. Free combination: Allows an item to be used soveral times in a proof, for example for the non-unit clauses of the program, which would be represented as items of the form $\left\langle X \hookleftarrow G_{1}^{y} \wedge \ldots \wedge G_{n}\right.$, frees $\rangle$.

The following table summarizes the propertics of these five combination schemes. Index 1 (II) is the index associated with the non-unit clause, Index 2 (IR) is associated with the unit clause, and $I 1 \star I 2$ is the result of combining the indices.

\begin{tabular}{|c|c|c|c|l|}
\hline & $\begin{array}{c}\text { Index 1 } \\
I 1\end{array}$ & $\begin{array}{c}\text { Indcx } 2 \\
I 2\end{array}$ & $\begin{array}{c}\text { Resull } \\
I 1 * I 2\end{array}$ & Nolc \\
\hline 1. & $X$ & $Y$ & $X \cup Y$ & $X \cap Y=\emptyset$ \\
\hline 2. & $X$ & $Y$ & $X \odot Y$ & \\
\hline 3. & $X+Y$ & $Y+Z$ & $X+Z$ & \\
& $Y+Z$ & $X+Y$ & $X+Z$ & \\
\hline 4. & $X-Y$ & $Y-Z$ & $X-Z$ & \\
\hline 5. & $X$ & 'free' & $X$ & \\
& 'free' & $X$ & $X$ & \\
\hline
\end{tabular}

In case 2 ("non-adjacent combination"), the indices $X$ and $Y$ consist of a set of string positions, and the operation $\odot$ is the union of these string positions, provided that no two string positions from $X$ and $Y$ do overlap.

In (2), the reduction rule is angmented to handle indices. $X \star Y$ denotes the combination of the indices $X$ and $Y$.

$$
\begin{gathered}
\langle X \leftarrow G \wedge \Omega, I 1\rangle \\
\left\langle G^{\prime} \leftarrow, I 2\right\rangle \\
\langle\sigma(X \leftarrow \Omega), I 1 * I 2\rangle
\end{gathered}
$$

With the use of indices, the lookup relation becomes a relation between goals and items. 'The following specification of the Iookup relation provides indexing according to string positions as in a chart parser (usable for combination schemes 2 , 3 , and 4 ).

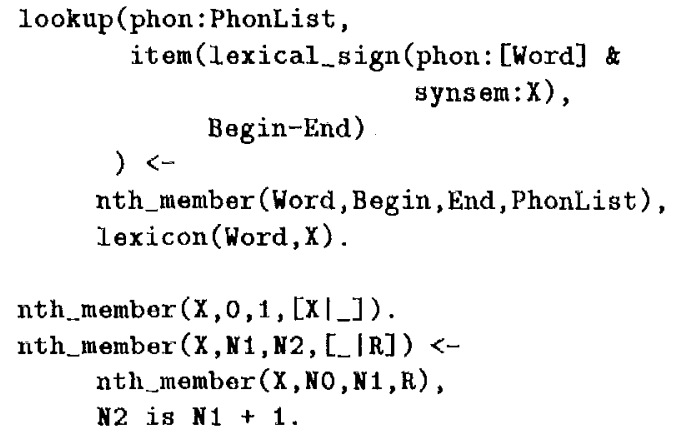

\section{$2.3 \quad$ Goal Types}

In constraint-based grammars there are some predicates that are not adequately dealt with by bottom-up liarley deduction, for example the Ilead Feature Principle and the Subcategorization Principle of Irrsg. The Head Feature Principle just unifies two variables, so that it can be executed at compile time and need not be called as a goal at runtime. The Subcategorization Principle involves an operation on lists (append/3 or delete/3 in different formalizations) that does not need bottom-11p processing, but can better be evaluated by top-down resolution if its arguments are sufficiently instantiated. Creating and managing items for these proofs is too much of a computational overhead, and, moreover, a proof may not terminate in the bottom-up case because infinitely many consequences may be derived from the base case of a recursively defined relation.

In order to deal with such goals, we associate the goals in the body of a clause with goal types. 'The goals that are relcvant for bottom-up Earley deduction are called wailing goals because they wait until they are activated by a unit clause that unifies with the goal." Whenever a unit clause is

\footnotetext{
${ }^{6}$ The other goal types are top-down goals (top-down
} 
combined with a non-unit clause all goals up to the first waiting goal of the resulting clause are proved according to their goal type, and then a new clause is added whose selected goal is the first waiting goal.

In the following inference rule for clauses with mixed goal types, $\Xi$ is a (possibly empty) sequence of goals without any waiting goals, and $\Omega$ is a (possibly empty) sequence of goals starting with a waiting goal. $\sigma$ is the most general unifier of $G$ and $G^{\prime}$, and the substitution $\tau$ is the solution which results from proving the sequence of goals छ.

$$
\begin{gathered}
\langle X \leftarrow G \wedge \Xi \wedge \Omega, I 1\rangle \\
\left\langle G^{\prime} \leftarrow, I 2\right\rangle \\
\langle\tau \sigma(X \leftarrow \Omega), I 1 \star I 2\rangle
\end{gathered}
$$

\subsection{Correctness and Completeness}

In order to show the correctness of the system, we must show that the scanning step only adds consequences of the program to the chart, and that any items derived by the inference rule are consequences of the program clauses. The former is easy to show because all clauses added by the scanning step are instances of program clauses, and the inference rule performs a resolution step whose correctness is well-known in logic programming. The other goal types are also proved by resolution.

There are two potential sources of incompleteness in the algorithm. One is that the scanning step may not add all the program clauses to the chart that are needed for proving a goal, and the other is that the indexing may prevent the derivation of a clause that is needed to prove the goal.

In order to avoid incompleteness, the scanning step must add all program clauses that are needed for a proof of the goal to the chart, and the combination of indices may only fail for inference steps which are useless for a proof of the goal. That

depth-first search), $x$-corner goals (which combine bottonup and top-down processing like left-corner or head-corner algorithms), Prolog goals (which are directly executed by Prolog for efficiency or side-effects), and chart goals which create a new, independent chart for the proof of the goal. Dörre [3] proposes a system with two goal types, namely trigger goals, which lead to the creation of items and other goals which don't. the lookup relation and the indexing scheme satisfy this property must be shown for particular grammar formalisms.

In order to keep the search space small (and finite to ensure termination) the scanning step should (ideally) add only those items that are needed for proving the goal to the chart, and the in dexing should be chosen in such a way that it excludes derived items that are useless for a proof of the goal.

\section{Best-First Search}

For practical NI applications, it is desirable to have a best-first search strategy, which follows the most promising paths in the search space first, and finds preferred solutions before the less preferred ones.

There are often situations where the criteria to guide the search are available only for the base cases, for example

- weighted word hypotheses from a speech recognizer

- readings for ambigous words with probabilities, possibly assigned by a stochastic tagger (cf. $[2]$ )

- hypotheses for correction of string errors which should be delayed [5]

Goals and clauses are associated with preference values that are intended to model the degrce of confidence that a particular solution is the 'correct' one. Unit clanses are associated with a numerical preference value, and non-unit clauses with a formula that determines how its preference value is computed from the preference values of the goals in the body of the clause. Preference values can (but need not) be interpreted as probabilities. $^{7}$

The preference values are the basis for giving priorities to items. For unit clauses, the priority is identified with the preference value. For non-unit clauses, where the preference formula may contain uninstantiated variables, the priority is the value of the formula with the free variables instantiated to the highest possible preference value (in case

\footnotetext{
${ }^{7}$ For further details and examples see [4] and [5].
} 
of an interpretation as probabilities: 1), so that the priority is equal to the maximal possible proference value for the clause. ${ }^{8}$

The implementation of best-first search does not combine new items with the chart immediately, but makes use of an agenda [8], on which new items are ordered in order of descending priority. The following is the algorithm for bottom-up bestfirst Earley deduction.

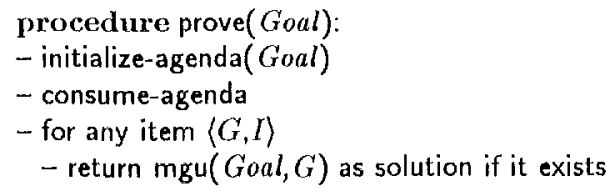

The algorithm is parametrized with respect to the relation lookup/ 2 and the choice of the indexing scheme, which are specific for different grammatical theories and directions of processing.

\footnotetext{
${ }^{8}$ There are also other methods for assigning priorities to iterns.
}

\section{Implementation}

The bottom-up Earley deduction algorithm described here has been implemented in Quintus Prolog as part of the GeID system. GeLD (Generalized Linguistic Deduction) is an extension of Prolog which provides typed feature descriptions and preference values as additions to the expressivity of the language, and partial evaluation, topdown, head-driven, and bottom-up Earley dedıction as processing strategies. Tests of the system with small grammars have shown promising results, and a medium-scale HPSG for German is presently being implemented in GeLD. The lookup relation and the choice of an indexing scheme must be specified by the user of the system.

\section{Conclusion and Future Work}

We have proposed bottom-up Earley deduction as a useful alternative to the top-down methods which require subsumption checking and restriction to avoid prediction loops.

The proposed method should be improved in two directions. The first is that the lookup predicate should not have to be specified by the user, but automatically inferred from the program.

The second problem is that all non-unit clauses of the program are added to the chart. The addition of non-unit clauses should be made dependent on the goal and the base cases in order to go from a purely bottom-up algorithm to a directed algorith $m$ that combines the advantages of top-down and bottom-np processing. It has been repeatedly noted $[8,17,1]$ that directed methods are more efficient than pure top-down or bottomup methods. However, it is not clear how well the directed methods are applicable to grammars which do not depend on concatenation and have no unique 'left corner' which should be connected to the start symbol.

It remains to be seen how bottom-up Farley deduction compares with (and can be combined with) the improved top-down Earley deduction of Dörre [3], Johnson [7] and Neumann [9], and to head-driven methods with well-formed substring tables [1], and which methods are best suited for which kinds of problems (e.g. parsing, generation, noisy input, incremental processing etc.). 


\section{References}

[1] Gosse Bouma and Gertjan van Noord. Headdriven parsing for lexicalist grammars: Experimental results. In $E A C L 93$, pages $71-80$, Utrecht, NL, 1993.

[2] Chris Brew. Adding preferences to CUF. In Jochen Dörre, editor, DYANA-2 Deliverable R1.2.A: Computational Aspects of Constraint-Based Linguistic Description I, pages 57-69. Esprit Basic Research Project $6852,1993$.

[3] Jochen Dörre. Generalizing Earley deduction for constraint-based grammars. In Jochen Dörre, editor, DYANA-2 Deliverable R1.2.A: Computational Aspects of Constraint-Based Linguistic Description I, pages 23 - 41. Esprit Basic Research Project 6852, 1993.

[4] Gregor Erbach. Using preference values in typed feature structures to exploit non-absolute constraints for disambiguation. In Harald Trost, editor, Feature Formalisms and Linguistic Ambiguity. Ellis-Horwood, 1993.

[5] Gregor Erbach. Towards a theory of degrees of grammaticality. In Carlos Martín-Vide, editor, Current Issues in Mathematical Linguistics. North-Holland, Amsterdam, to appear. Also published as CLAUS Report 34, Universität des Saarlandes, 1993.

[6] Dale Douglas Gerdemann. Parsing and Generation of Unification Grammars. PhD thesis, University of Illinois at UrbanaChampaign, 1991. Cognitive Science technical report CS-91-06 (Language Series).

[7] Mark Johnson. Memoization in constraint logic programming. Department of Cognitive Science, Brown University. Presented at the 1st International Conference on Constraint Programming, Newport, Rhode Island; to appear in the proceedings, 1993.

[8] Martin Kay. Algorithm schemata and data structures in syntactic processing. Technical Report CSL-80-12, XEROX PARC, Palo Alto, CA, 1980.
[9] Günter Neumann. A Uniform Tabular Algorithm for Natural Language Parsing and Generation and its Use within Performancebased Methods. PhD thesis, University Saarbrücken. forthcoming.

[10] Fernando C.N. Pereira and David H.D. Warren. Parsing as deduction. In ACL Proceedings, 21st Annual Meeting, pages 137-144, 1983 .

[11] Carl Pollard. Generalized Context-Free Grammars, Head Grammars, and Natural Language. PhD thesis, Stanford, 1984.

[12] Mike Reape. A theory of word order and discontinuous constituency in West Germanic. In F. Engdahl and M. Reape, editors, Parametric Variation in Germanic and Romance: Preliminary Investigations, pages 25-40. ESPRIT Basic Research Action 3175 Dyana, Deliverable R1.1.A, 1990.

[13] Stuart M. Shieber. A uniform architecture for parsing and generation. In Proceedings of the 12th International Conference on Computational Linguistics (COLING), Budapest, 1988.

[14] Gertjan van Noord. Reversibility in Natural Language Processing. PhD thesis, Rijksuniversiteit Utrecht, NL, 1993.

[15] K. Vijay-Shanker, David J. Weir, and Aravind $\mathrm{K}$. Joshi. Characterizing structural descriptions produced by various grammatical formalisms. In 25th Annual Meeting, pages 104-111, Stanford, CA, 1987. Association for Computational Linguistics.

[16] David J. Weir. Characterizing Mildly Context-Sensitive Grammar Formalisms. PhD thesis, Department of Computer and Information Science, University of Pennsylvania, 1988.

[17] Mats Wirén. A comparison of rule-invocation strategies in context-free chart parsing. In ACL Proceedings, Third European Conference, pages 226-235, 1987. 